\title{
Evaluation of Elastic Lift for Facial Rejuvenation
}

\author{
Moon Seok Kang ${ }^{1}$, Jin Su Shin ${ }^{2}$, \\ Seung Min Nam², Eun Soo Park ${ }^{2}$ \\ ${ }^{1}$ Garosu Plastic Surgery Clinics, Seoul; \\ ${ }^{2}$ Department of Plastic and Reconstructive \\ Surgery, Soonchunhyang University \\ College of Medicine, Bucheon, Korea
}

Supplemental digital content is available for this article. Direct URL citations appear in the printed text and are provided in both the HTML and PDF versions of this article on the journal's Web site (www.e-aaps.org).

This work was supported by the Soonchunhyang University Research Fund.

No potential conflict of interest relevant to this article was reported.
Background Thread lift is a minimally invasive procedure for facial rejuvenation, but complications of thread lift have been reported. The purpose of this study was to evaluate outcomes and safety associated with elastic lift in relation to midface and upper mandibular region lifting.

Methods Thirty-eight patients with sagging and laxity of the facial skin treated with elastic lift from September 2015 to October 2015 were enrolled in this study. Treatment was performed following the manufacturer's recommended protocol that called for 300 treatment lines. To evaluate elastic lift safety, a retrospective chart review was conducted. We used an automatic skin diagnosis system to evaluate the efficacy of elastic lift.

Results Thirty-seven patients were female, and one patient was male. The mean age of the patients was 40.4 years, and their mean follow-up period was 3 months. There were no major complications manifesting in removal of the elastic thread during the followup period. The median skin laxity grade score was $5(3-6)$ before surgery; the median skin laxity grade score was $3(2-4) 3$ months post-surgery, resulting in a statistically significant difference in skin grade score $(\mathrm{P}<0.01)$.

Conclusions Facial rejuvenation using elastic lift is an effective and safe procedure in select patients with midface ptosis and moderate jowls.

Keywords Aging face, Thread lift, Facial rejuvenation

\section{INTRODUCTION}

Facial aging is the progression of three processes: skin and soft tissue laxity, superficial skin changes, and volume loss [1-5]. Understanding these processes is essential when intending to achieve natural results for the aging face.

Since Mitz and Peyronie [6] defined the superficial musculoaponeurotic system (SMAS), facial rejuvenation techniques have evolved from subcutaneous rhytidectomy to soft tissue repositioning and SAMS lift [7]. However, these invasive facelifts are elaborate and complicated procedures that require a long recovery time $[8,9]$. The use of barbed sutures to lift various ptotic areas of the face can be a

Received: Jan 21, 2016 Revised: Feb 1, 2016 Accepted: Feb 2, 2016 Correspondence: Seung Min Nam Department of Plastic and Reconstructive, Soonchunhyang University Bucheon Hospital, 170 Jomaru-ro, Wonmi-gu, Bucheon 14584, Korea. E-mail: zodiac1003@naver.com

Copyright @ 2016 The Korean Society for Aesthetic Plastic Surgery.

This is an Open Access article distributed under the terms of the Creative Commons Attribution Non-Commercial License (http://creativecommons.org/licenses/by-nc/3.0/) which permits unrestricted non-commercial use, distribution, and reproduction in any medium, provided the original work is properly cited. www.e-aaps.org good alternative for more invasive procedures [8,10-13]. The thread lift shows satisfactory results with minimal scarring, rapid recovery, and minimal complications [11-13]. Nevertheless, complications of thread lift have been reported, including facial asymmetry, recurrence, and the relative lack of efficacy [14].

In this study, we analyzed a new type of thread lift: elastic lift that uses elastic thread (Elasticum ${ }^{\circledR}$, Korpo SRL, Genova, Italy). The purpose of this study was to evaluate outcomes and safety associated with elastic lift in relation to midface and upper mandibular region lifting.

\section{METHODS}

Of the patients who underwent elastic lift between September 2015 and October 2015, 38 patients who could be observed and followed for longer than 3 months were enrolled in this study. Patients who had active systemic or local infections, local skin diseases that might alter wound healing, a history of psychiatric illness, or soft tissue augmentation materials were excluded from the study.

Medical charts and operative records were reviewed retrospectively to evaluate postoperative outcomes and complications. The 
study conformed to the Declaration of Helsinki. Written consent was obtained from each patient for both the surgery and publication of the results.

\section{Elastic thread}

The elastic thread consists of nonabsorbable suture material (Elasticum $^{\circledR}$ ) and a two-tipped long needle (Jano needle ${ }^{\circledR}$ ). The nonabsorbable elastic suture material, made of silicone and sheathed with polyester, is designed for the suspension and traction of tissues. The two-tipped long needle enables the elastic thread to be implanted without blunt dissection of the tissues. The elastic suture material is attached to the center portion of the needle, and there are scale marks on both sides of the needle.

\section{Surgical procedure}

\section{Preoperative design}

The preoperative design was performed under an upright sitting position. We evaluated the mobility of the midface. We drew two points (A and B) at the lower temporal region within the hairline located $2 \mathrm{~cm}$ above the root of the zygomatic arch. The distance between $A$ and $B$ was about $2 \mathrm{~cm}$. The line that connects the lateral margin of the alar base and the root of the ear lobe was drawn. We drew two points ( $\mathrm{C}$ and $\mathrm{D})$ at the lower mandibular region located $1.5 \mathrm{~cm}$ below that line (Fig. 1).

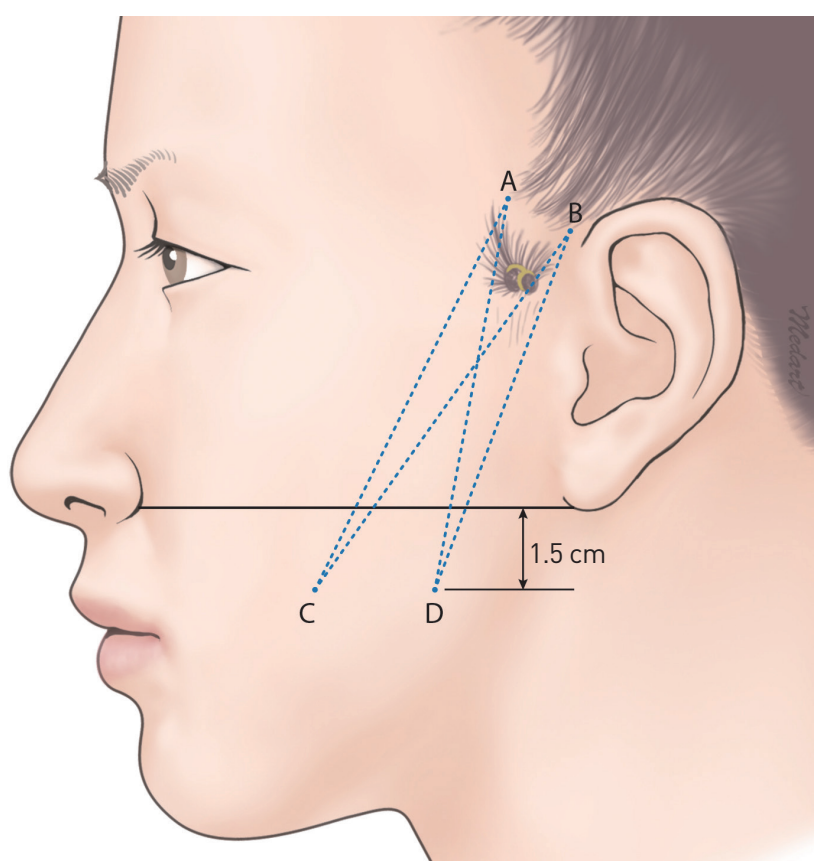

Fig. 1. Schematic drawing of the preoperative design. Points $A$ and $B$ were drawn at the lower temporal region within the hairline. The line that connects the lateral margin of the alar base and the root of ear lobe was drawn. We drew points $C$ and $D$ at the lower mandibular region located $1.5 \mathrm{~cm}$ below that line.

\section{Surgical technique}

All surgeries were performed under conscious sedation. The scalp at both incision sites were infiltrated with 2\% lidocaine and 1:100,000 epinephrine. We began by performing two stab incisions at the lower temporal region. Sharp Metzenbaum scissors were used to dissect down to the temporal fascia. An Adson-Brown forceps was used at the skin edge as the Jano needle ${ }^{\circledast}$ was inserted at point A. The needle was then passed through the subcutaneous tissue layers with great care not to injure the dermis. The needle was extracted up to the last depth-mark at point $\mathrm{C}$, and the elastic thread was pulled through. The needle was rotated towards point $\mathrm{B}$ with the posterior tip now becoming anterior. The needle was passed through the subcutaneous layers. The needle was extracted at point $B$ until about $5 \mathrm{~mm}$ of the tip remained in the tissue and the elastic thread was pulled through. The needle was rotated towards point $\mathrm{A}$ with the posterior tip now becoming anterior. The needle was passed through the deep temporal fascia. The needle was extracted at point $A$ and the elastic thread was pulled through. The elastic thread was then placed securely under tension and knotted (See Video, Supplemental Digital Content 1). The same procedure was performed $(B \rightarrow D \rightarrow A \rightarrow B)$. When the dermis was captured at point $C$ or $D$, the 18-gauze needle was used for release. The two stab incisions at the lower temporal region were sutured with No. 5-0 nylon. The head was wrapped with a 3 -inch elastic bandage and the patient was taken to the recovery room.

\section{Evaluation of outcomes}

We evaluated the patients using an automatic skin diagnosis system (A-One Lite ${ }^{\circledR}$; BOMTECH Electronics Co., Seoul, Korea) before surgery and 3 months after surgery. The automatic skin diagnosis system evaluates skin laxity using a skin scanner. The sagging and laxity of patients were graded from 1 to 6 using the automatic skin diagnosis system. A high skin grade score means severe sagging and laxity of patient skin.

\section{Statistical analysis}

Statistical analyses were performed using SPSS version 20.0 (SPSS Inc., Chicago, IL, USA). The Wilcoxon signed rank test was used to compare the grade scores of patients before surgery and 3 months after surgery. A P-value less than 0.05 was considered statistically significant.

\section{RESULTS}

Of the 38 patients treated using elastic lift, 37 patients were female and 1 patient was male. The mean age of the patients was 40.4 years (range, 28-53 years), and their mean follow-up period was 3 months (range, 3-4 months) (Table 1). Five patients immediately presented with mild post-surgical asymmetry, which was corrected by slight compression therapy. In 11 patients, an unnatural puckering ap- 
Table 1. Patients characteristics

\begin{tabular}{lc}
\hline Characteristic & Value \\
\hline Sex (female:male) & $37: 1$ \\
Mean age (range) & $40.4(28-53)$ \\
\hline
\end{tabular}

pearance was released by abrupt gentle massage.

There were no major complications manifesting the removal of elastic thread during the follow-up period (Fig. 2-5). The most frequent minor complication was the presence of ecchymosis in 24 patients (63\%). This ecchymosis was protracted for a maximum of
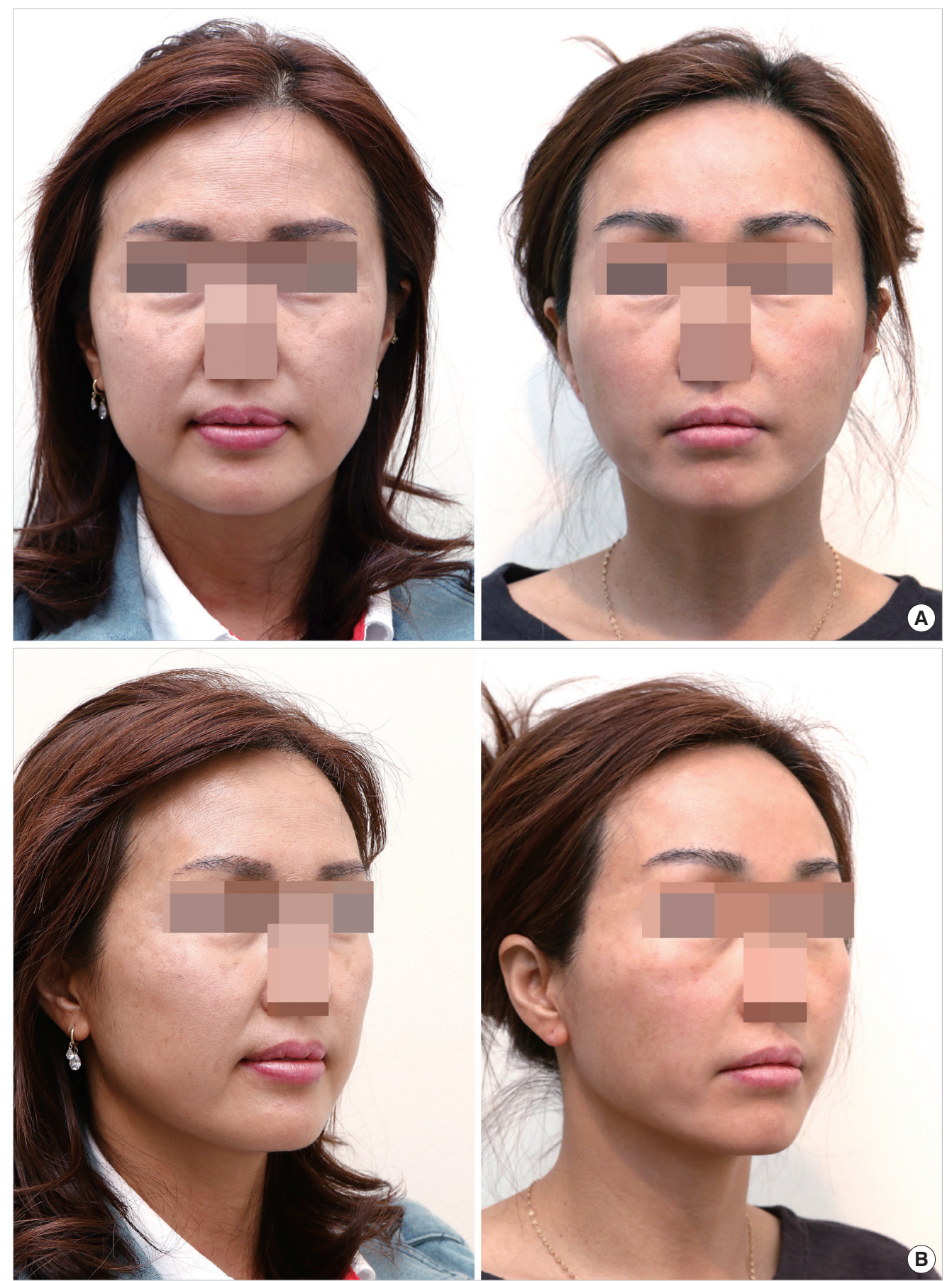

Fig. 2. A 52-year-old female patient with moderate skin sagging and wrinkling. (A) Preoperative frontal photographic finding. The patient was examined by an automatic skin diagnosis system, and the skin grade score was assessed (Left). Three months after surgery, the skin grade score was assessed (Right). (B) Preoperative oblique photographic finding (Left). Postoperative oblique photographic finding (Right). 
3 weeks and did not demand any treatment. In eight patients (21\%), slight bleeding occurred immediately after the insertion of the thread in the subcutaneous layer. It was treated by mechanical compression. All patients were able to return to their daily activities 3 days after surgery.
The median skin laxity grade score was 5 (3-6) before surgery; the median skin laxity grade score was 3 (2-4) 3 months after surgery (Fig. 6), resulting in a statistically significant difference in skin grade score $(\mathrm{P}<0.01)$.

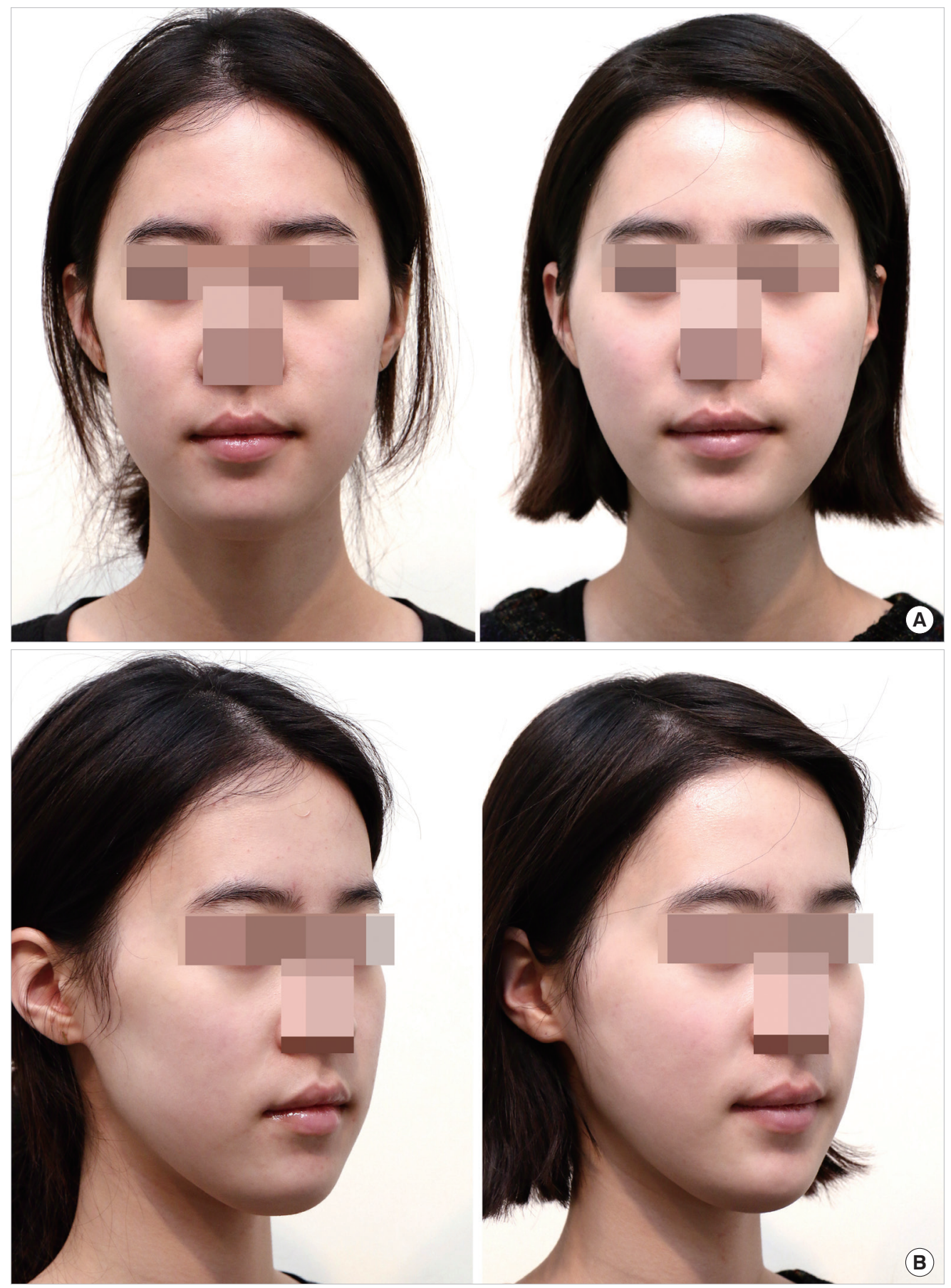

Fig. 3. A 28-year-old female patient with mid skin sagging. (A) Preoperative frontal photographic finding. The patient was examined by an automatic skin diagnosis system, and the skin grade score was assessed (Left). Three months after surgery, the skin grade score was assessed (Right). (B) Preoperative oblique photographic finding (Left). Postoperative oblique photographic finding (Right). 

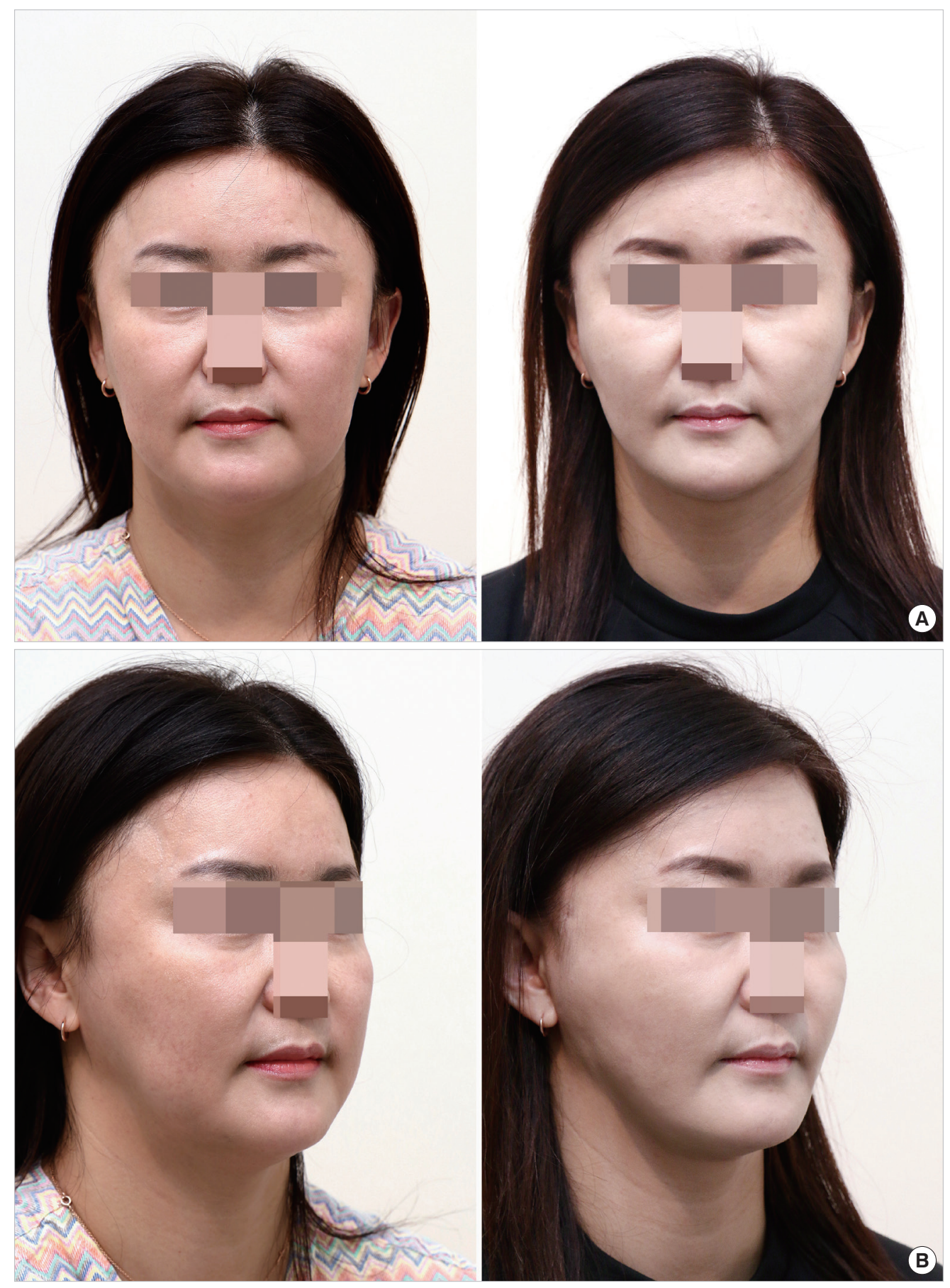

Fig. 4. A 50-year-old female patient with moderate skin sagging and wrinkling. (A) Preoperative frontal photographic finding. The patient was examined by the automatic skin diagnosis system, and the skin grade score was assessed (Left). Three months after surgery, the skin grade score was assessed (Right). (B) Preoperative oblique photographic finding (Left). Postoperative oblique photographic finding (Right).

\section{DISCUSSION}

Recently, there has been a growing trend for patients to pursue minimally invasive facial rejuvenation with a low risk of complications and rapid recovery time [15]. Numerous minimally invasive procedures have been used to improve the results of the aging face [16]. Since Sulamanidze introduced a minimally invasive procedure for lifting and rejuvenating facial tissues using bidirectional barbed sutures, various minimally invasive facial rejuvenation procedures, such as Woffles thread lifting, Waptos suture lifting, Isse 


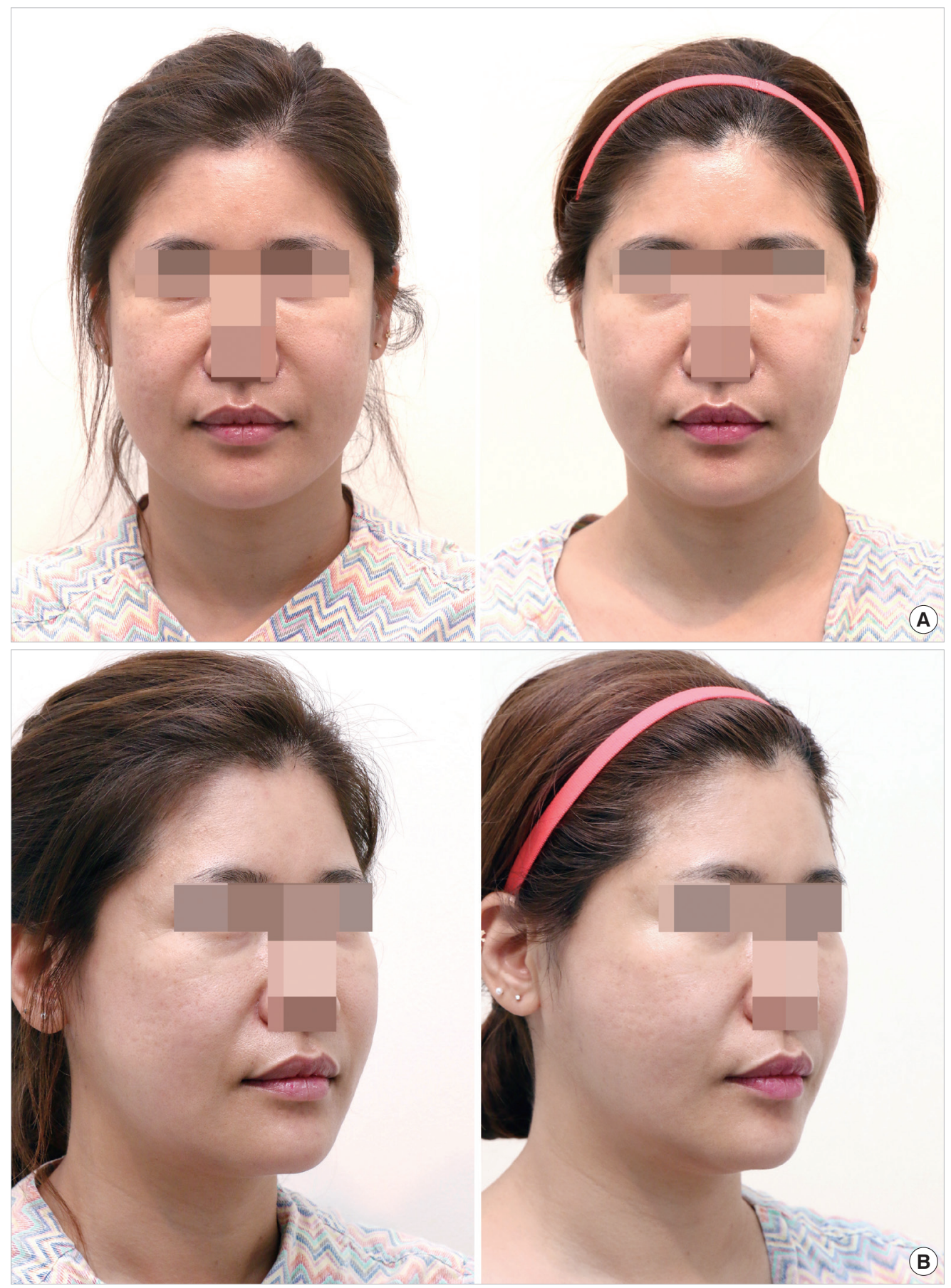

Fig. 5. A 46-year-old female patient with moderate skin sagging and wrinkling. (A) Preoperative frontal photographic finding. The patient was examined by an automatic skin diagnosis system, and the skin grade score was assessed (Left). Three months after surgery, the skin grade score was assessed (Right). (B) Preoperative oblique photographic finding (Left). Postoperative oblique photographic finding (Right).

unidirectional barbed threads lifting, and silhouette lifting, have been developed $[11,13,17,18]$. These minimally invasive facial rejuvenation procedures share a common concept that ptotic facial soft tissues are suspended with barbed suture material [10].

Complications such as thread extrusion, skin dimpling, hemor- rhage, ecchymosis, erythema, and persistent pain after performing thread lifts have been reported [19-21]. Abraham et al. [10] argued that there were two reasons that the thread lift is ineffective for facial rejuvenation: It does not provoke any volumetric change and it repositions the soft tissues in a superficial plane. Stuzin et al. [22] 


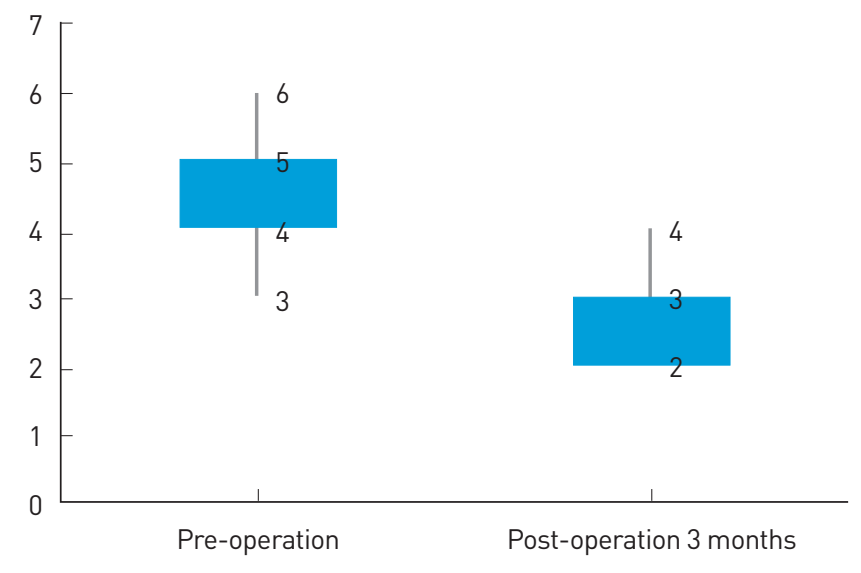

Fig. 6. Median skin laxity grade scores before and after surgery.

argued that the thread suture could tear the SMAS when the thread sutures are inserted the SMAS layer under tension .

Elastic lift has three advantages over existing thread lift. First, nonabsorbable suture material of elastic thread is sheathed with polyester. The polyester surface is not barbed but braided. Huggins et al. [23] reported that the connective tissue that continues into the interstices of the braided suture has characteristics of a ligament. Therefore, elastic thread is a suitable fixation material for maintaining long-term elevation of ptotic facial soft tissues. Second, when removal of the elastic thread is required within 2 weeks post-surgery, elastic thread can easily be removed because the nonabsorbable suture material is not barbed. Third, elastic lift induces better facial volumetric changes. The mechanism of existing thread lift is traction and suspension of soft tissue using barbed suture material, whereas that of elastic lift is the repositioning of soft tissue using the elasticity of silicone.

However, all aging faces cannot be treated by elastic lift. Elastic lift should not be used for redundant skin excision. Therefore, patients with severe skin sagging cannot be treated by elastic lift. When patients with a square face were treated by elastic lift, the post-surgical width of the face was wider than before surgery, because the soft tissue of the lower face is translocated by elastic lift in an upper and lateral direction. Based on our clinical experience, when we perform elastic lift, patient selection is very important. When elastic lift is performed, patients with a long and narrow face show better results than patients with a wide and square face, who often have complications, such as increased facial width, which may occur after surgery. Patients with cheek dropping caused by zygoma reduction or fracture are also good candidates for elastic lift.

Our study has limitations. First, we did not include patients with severe skin sagging. Elastic lift does not include redundant skin excision. Therefore, we recommend a more invasive procedure for these patients. Second, the post-surgical results were evaluated with an automatic skin diagnosis system, but the reliability of this system has not been established. Therefore, errors between the auto- matic skin diagnosis system and realistic skin wrinkling may occur. Third, our study has a short follow-up period. We agree that longer-term follow-up studies are needed to prove a definite effect of elastic lift. Despite these limitations, the significance of our study is that it is first study in which the results of elastic lift were evaluated by objective analyses.

\section{CONCLUSIONS}

Although further studies with long-term follow-up periods are needed to determine whether elastic lift provides long-lasting satisfying results, facial rejuvenation using elastic lift is an effective and safe procedure in select patients.

\section{PATIENT CONSENT}

Patients provided written consent for the use of their images.

\section{REFERENCES}

1. Donofrio LM. Fat distribution: a morphologic study of the aging face. Dermatol Surg 2000;26:1107-12.

2. Muzaffar AR, Mendelson BC, Adams WP Jr. Surgical anatomy of the ligamentous attachments of the lower lid and lateral canthus. Plast Reconstr Surg 2002;110:873-84; discussion 97-911.

3. Pessa JE, Zadoo VP, Mutimer KL, et al. Relative maxillary retrusion as a natural consequence of aging: combining skeletal and soft-tissue changes into an integrated model of midfacial aging. Plast Reconstr Surg 1998;102:205-12.

4. Williams EF 3rd, Smith SP Jr. Minimally invasive midfacial rejuvenation: combining thread-lift and lipotransfer. Facial Plast Surg Clin North Am 2007;15:209-19, vii.

5. Yousif NJ. Changes of the midface with age. Clin Plast Surg 1995;22: 213-26.

6. Mitz V, Peyronie M. The superficial musculo-aponeurotic system (SMAS) in the parotid and cheek area. Plast Reconstr Surg 1976;58:80-8.

7. Paul MD, Calvert JW, Evans GR. The evolution of the midface lift in aesthetic plastic surgery. Plast Reconstr Surg 2006;117:1809-27.

8. Savoia A, Accardo C, Vannini F, et al. Outcomes in thread lift for facial rejuvenation: a study performed with happy lift revitalizing. Dermatol Ther (Heidelb) 2014;4:103-14.

9. Chaffoo RA. Complications in facelift surgery: avoidance and management. Facial Plast Surg Clin North Am 2013;21:551-8.

10. Abraham RF, DeFatta RJ, Williams EF 3rd. Thread-lift for facial rejuvenation: assessment of long-term results. Arch Facial Plast Surg 2009; 11:178-83.

11. Isse NG, Fodor PB. Elevating the midface with barbed polypropylene sutures. Aesthet Surg J 2005;25:301-3.

12. Ruff G. Technique and uses for absorbable barbed sutures. Aesthet Surg J 2006;26:620-8. 
13. Wu WT. Barbed sutures in facial rejuvenation. Aesthet Surg J 2004;24: 582-7.

14. Sapountzis S, Kim JH, Li TS, et al. Successful treatment of thread-lifting complication from APTOS sutures using a simple MACS lift and fat grafting. Aesthetic Plast Surg 2012;36:1307-10.

15. Atiyeh BS, Dibo SA, Costagliola M, et al. Barbed sutures "lunch time" lifting: evidence-based efficacy. J Cosmet Dermatol 2010;9:132-41.

16. Rachel JD, Lack EB, Larson B. Incidence of complications and early recurrence in 29 patients after facial rejuvenation with barbed suture lifting. Dermatol Surg 2010;36:348-54.

17. Suh DH, Jang HW, Lee SJ, et al. Outcomes of polydioxanone knotless thread lifting for facial rejuvenation. Dermatol Surg 2015;41:720-5.

18. Park TH, Seo SW, Whang KW. Facial rejuvenation with fine-barbed threads: the simple Miz lift. Aesthetic Plast Surg 2014;38:69-74.
19. Lee S, Isse N. Barbed polypropylene sutures for midface elevation: early results. Arch Facial Plast Surg 2005;7:55-61.

20. Lycka B, Bazan C, Poletti E, et al. The emerging technique of the antiptosis subdermal suspension thread. Dermatol Surg 2004;30:41-4; discussion 4.

21. Stark GB, Bannasch H. The "golden thread lift": radiologic findings. Aesthetic Plast Surg 2007;31:206-8.

22. Stuzin JM, Baker TJ, Baker TM. Refinements in face lifting: enhanced facial contour using vicryl mesh incorporated into SMAS fixation. Plast Reconstr Surg 2000;105:290-301.

23. Huggins RJ, Freeman ME, Kerr JB, et al. Histologic and ultrastructural evaluation of sutures used for surgical fixation of the SMAS. Aesthetic Plast Surg 2007;31:719-24. 